\title{
Role of rotational energy component in the dynamics of ${ }^{16} \mathrm{O}+{ }^{198} \mathrm{Pt}$ reaction
}

\author{
Manoj K. Sharma ${ }^{1, \text { a }}$, Rajni ${ }^{1}$, and Deepika Jain ${ }^{2}$ \\ 1 School of Physics and Materials Science, Thapar University, Patiala, Punjab, India \\ 2 Department of Physics, Mata Gujri College, Fatehgarh sahib, Punjab, India
}

\begin{abstract}
The role of rotational energy is investigated in reference to the dynamics of ${ }^{16} \mathrm{O}+{ }^{198} \mathrm{Pt} \rightarrow{ }^{214} \mathrm{Rn}^{*}$ reaction using the sticking $\left(I_{\mathrm{S}}\right)$ and the non-sticking $\left(I_{\mathrm{NS}}\right)$ limits of moment of inertia within the framework of dynamical cluster decay model. The decay barrier height and barrier position get significantly modified for the use of sticking or non-sticking choice, which in turn reproduce the evaporation residue and the fusion-fission cross-sections nicely by the $I_{\mathrm{S}}$ approach, while the $I_{\mathrm{NS}}$ approach provides feasible addressal of data only for evaporation residue channel. Moreover, the fragmentation path of decaying fragments of ${ }^{214} \mathrm{Rn}^{*}$ compound nucleus gets influenced for different choices of moment of inertia. Beside this, the role of nuclear deformations i.e. static, dynamic quadurpole $\left(\beta_{2}\right)$ and higher order static deformation up to $\beta_{4}$ are duly investigated for both choices of the moment of inertia.
\end{abstract}

\section{Introduction}

The study of reaction dynamics in low-energy heavy-ion induced reactions has been of immense interest in recent years. It has been observed that when lighter $\mathrm{Z}$ projectiles $(\mathrm{Z} \leq 10)$, e.g., ${ }^{12} \mathrm{C},{ }^{16} \mathrm{O}$, and ${ }^{20} \mathrm{Ne}$ with energy below $10 \mathrm{MeV} /$ nucleon, interact with the medium and heavy mass target, having energy slightly above the Coulomb barrier, then the fusion process (other than incomplete fusion) may be considered as one of the dominant reaction mechanism [1]. In the fusion reaction process, the projectile gets amalgamated with the target nucleus along all the degrees of freedom and linear momentum gets transferred from projectile to target leading to fully equilibrated excited compound nucleus $(\mathrm{CN})$, having high excitation energy and angular momentum. The compound nuclear reactions are particularly useful in the synthesis of new elements and to study the structure and properties of excited nuclear systems.

For heavy compound systems formed in the fusion reactions, fission becomes a significant exit channel, however for lighter nucleus, the smaller fragments such as evaporation residues (ER) form the major decay mode. In literature, there are large numbers of theoretical models, which use variety of the nuclear potentials and explain relevant features of the fusion-fission (ff) dynamics. In theoretical study of heavy ion induced reactions, the long range (Coulomb and centrifugal) and the short range (proximity) interactions $[2,3]$ play a very important role. During the past few years, significant amount of work has been done to understand the specific contribution of various interaction potentials in the heavy ion induced dynamics [4-8]. So, it is of further interest to study the effect of long range interaction potential (centrifugal part) in the decay processes (evaporation residue and

\footnotetext{
a e-mail: msharma@thapar.edu
}

fission) of $\mathrm{CN}$. In the present work, centrifugal potential is investigated within two limits of momentum of inertia such as (i) Sticking limit $I_{\mathrm{S}}$ [9] (ii) Non Sticking limit $I_{\mathrm{NS}}$ [6]. There are various hypothesis other than these two limits such as rolling and sliding conditions. The sticking limit $\left(I_{\mathrm{S}}\right)$ is more appropriate [6] for the use of proximity potential with neck-length surface $\leq 2 \mathrm{fm}$, which has the consequences that, the limiting angular momentum is much larger in this case as compared to $I_{\mathrm{NS}}$ limit where lesser number partial waves contribute towards cross-sections. The previous studies $[7,8,10,11]$ suggests that, both $I_{\mathrm{S}}$ and $I_{\mathrm{NS}}$ limits are equally good to study evaporation residue process, although, in fission process $I_{\mathrm{S}}$ seems to perform better $[6,11]$. These observations are for independent addressal of ER and fission paths governed in independent reactions induced via heavy ion collisions. In order to generalize this aspect, we intended to carry out the comprehensive decay analysis of a heavy ion reaction exhibiting evaporation residue and fission paths over a common range of center of mass energy.

Here, we have investigated the dynamics of ${ }^{214} \mathrm{Rn}^{*}$ compound nucleus, which is formed via ${ }^{16} \mathrm{O}+{ }^{198} \mathrm{Pt}$ reaction. The experiment was performed at 15 UD Pelletron accelerator facility of the IUAC [12], in order to measure the evaporation residue excitation functions over a wide range of center of mass energies. In an another experiment [13], the fission cross-sections of ${ }^{16} \mathrm{O}+{ }^{198} \mathrm{Pt}$ reaction were also measured. As mentioned above, the present interest is to study the effect of $I_{\mathrm{S}}$ and $I_{\mathrm{NS}}$ approaches for evaporation residue (ER) and fusion-fission (ff) paths governed in the reaction pertains to same target projectile combination. The comprehensive study of $I_{\mathrm{S}}$ and $I_{\mathrm{NS}}$ for excited ${ }^{214} \mathrm{Rn}^{*}$ compound nucleus is carried out using the dynamical cluster decay model (DCM) $[14,15]$. Beside this, the effect of lower (up to $\beta_{2}$ ) as well as higher order deformations $\left(\beta_{4}\right)$ are incorporated within $I_{\mathrm{S}}$ and $I_{\mathrm{NS}}$ limit of moment of inertia for the 
system under consideration. The optimum orientation angles $\theta^{\text {opt }}$ are chosen for $\beta_{2}$ deformed nuclei [16], however for $\beta_{4}$ deformed nuclei, compact orientations $\theta^{c}$ [17] are considered. In addition to this, the effect of temperature dependent deformation $\left(\beta_{2}(\mathrm{~T})\right)$ in reference to the sticking and non-sticking limit of moment of inertia is also worked out. The present study reveal that, the fragmentation path of decaying fragments is significantly influenced within both choices of the moment of inertia. The experimental data of evaporation residue and fusionfission is reproduced by taking only parameter of model known as neck-length parameter $\Delta \mathrm{R}$. It is anticipated from the calculations that, DCM based evaporation residue cross-sections seem in good comparison with experimental data for both $I_{\mathrm{S}}$ and $I_{\mathrm{NS}}$ moment of inertia, for all choices of deformations, however fusion-fission data is fitted only within $I_{\mathrm{S}}$ limit by opting higher order static deformations.

The paper is organized as follow: Brief methodology used for calculations is discussed in Sect. 2. Calculations and results are described in Sect. 3 and finally the outcome is summarized in Sect. 4.

\section{The methodology}

The dynamical cluster decay model (DCM) [14,15] writes the potential in terms of the mass and charge asymmetry coordinates $\eta=\left(A_{1}-A_{2}\right) /\left(A_{1}+A_{2}\right)$ [and $\left.\eta_{Z}=\left(Z_{1}-Z_{2}\right) /\left(Z_{1}+Z_{2}\right)\right]$, relative separation $\mathrm{R}$, the multipole deformations $\beta_{\lambda \mathrm{i}}$ and orientations $\theta_{i}(i=1,2)$ of two nuclei in the same plane between principal plane of two nuclei as

$$
\begin{aligned}
V(\eta, T)= & \sum_{i=1}^{2} V_{L D M}\left(A_{i}, Z_{i}, T\right)+\sum_{i=1}^{2} \delta U \exp \left(-\frac{T^{2}}{T_{0}^{2}}\right) \\
& +V_{C}\left(R, Z_{i}, \beta_{\lambda i}, \theta_{i}, T\right)+V_{N}\left(R, A_{i}, \beta_{\lambda i}, \theta_{i}, T\right) \\
& +V_{\text {rot }}\left(R, A_{i}, \beta_{\lambda i}, \theta_{i}, T\right) .
\end{aligned}
$$

Here, $V_{\mathrm{LDM}}$ is the T-dependent liquid drop model energy of Davidson et al. [18] and $\delta U$ is the "empirical" shell corrections of Myers and Swiatecki [19]. $V_{\mathrm{C}}$ and $V_{\mathrm{N}}$ are the T-dependent Coulomb and nuclear proximity potentials, respectively, and $V_{\text {rot }}$ represents the angular momentum dependent term.

The relation between rotational energy, angular momentum and related moment of inertia is given by,

$$
V_{r o t}\left(R, A_{i}, \beta_{\lambda i}, \theta_{i}, T\right)=\frac{\hbar^{2} \ell(\ell+1)}{2 I(T)} .
$$

Where $I(T)$ represents the moment of inertia of the dinuclear system and can be calculated by various methods, but in the present study two approaches are adopted: (i) Sticking limit, $\quad I=I_{\mathrm{S}}=\mu \mathrm{R}^{2}+\frac{2}{5} \mathrm{~A}_{1} \mathrm{mR}_{1}^{2}\left(\alpha_{1}\right)+$ $\frac{2}{5} A_{2} \mathrm{mR}_{2}^{2}\left(\alpha_{2}\right)$ (ii) non-sticking limit, $\mathrm{I}=I_{\mathrm{NS}}=\mu \mathrm{R}^{2}$. This use of reduced mass alone in latter expression corresponds to the supposition that the emission of the fragment is prompt.

The temperature effects in fragmentation potential (Eq. (1)) is introduced by scaling the radii of the two nuclei, as

$$
R_{i}\left(\alpha_{i}, T\right)=R_{0 i}(T)\left[1+\sum_{\lambda} \beta_{\lambda i} Y_{\lambda}^{(0)}\left(\alpha_{i}\right)\right]
$$
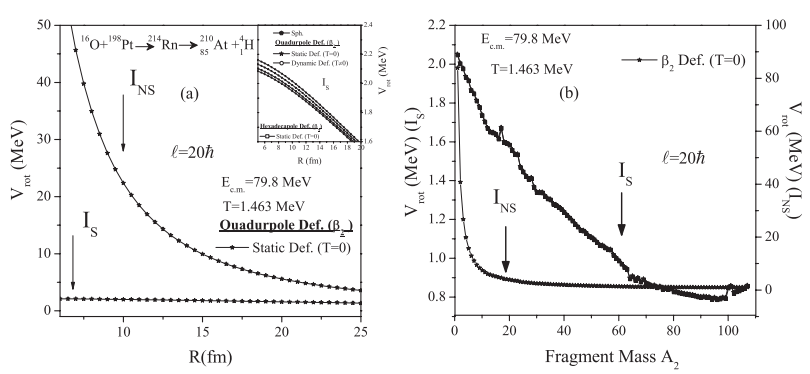

Figure 1. Variation of rotational energy $V_{\text {rot }}$ (or $V_{\ell}$ ) as a function of (a) internuclear distance $\mathrm{R}$ and (b) fragment Mass $\mathrm{A}_{2}$, plotted for ${ }^{16} \mathrm{O}+{ }^{198} \mathrm{Pt}$ reaction via both choices of moment of inertia at fixed $\ell$-value. The inset in (a) shows the behavior of $I_{S}$ with all spherical, static deformation $\left(\beta_{2}(0)\right)$, dynamic deformation $\left(\beta_{2}(\mathrm{~T})\right)$ and static hexadecupole deformation $\beta_{4}(0)$.

The deformations added here are temperature independent or static deformations $\beta_{\lambda_{\mathrm{i}}}(0)$. Since at higher excitation energies, the static deformation start approaching zero $[20,21]$, therefore the deformation parameter needs to be temperature dependent [20-22], termed as dynamic deformation $\beta_{\lambda \mathrm{i}}(\mathrm{T})$ by using relation

$$
\beta_{\lambda i}(T)=\exp \left(-\frac{T}{T_{0}}\right) \beta_{\lambda i}(0), i=(1,2),
$$

where $T_{0}$ is the temperature of the nucleus at which shell effects start to vanish $\left(T_{0}=1.5 \mathrm{MeV}\right)$ [22].

\section{Calculations and results}

We study the effect of rotational energy $V_{\text {rot }}$ in the decay path of ${ }^{214} \mathrm{Rn}^{*}$ compound nucleus formed in ${ }^{16} \mathrm{O}+{ }^{198} \mathrm{Pt}$ reaction via both modes of the moment of inertia i.e. the sticking $I_{\mathrm{S}}$ and the non-sticking $I_{\mathrm{NS}}$, which in turn influence the barrier characteristics and fragmentation path. Figure 1(a) shows the variation of centrifugal potential $\left(V_{\text {rot }}\right)$ as a function of internuclear distance R and, Fig. 1(b) depict the same as a function of fragment mass $A_{2}$ at a fixed temperature $\mathrm{T}=1.463 \mathrm{MeV}$ (equivalently, $E_{\text {c.m. }}=79.8 \mathrm{MeV}$ ) for the decay of ${ }^{214} \mathrm{Rn}^{*} \mathrm{CN}$ using quadurpole static deformation only. It is observed from Fig. 1(a) that, a steep fall of $V_{\text {rot }}(\mathrm{R})$ is observed on using $I_{\mathrm{NS}}$ limit up to the interaction range of $10 \mathrm{fm}$, however after $15 \mathrm{fm}$, it starts saturating. On the other hand, $V_{\text {rot }}$ is decreasing very slowly for the use of $I_{S}$ limit of moment of inertia. In order to look for the possible role of deformations and orientations, the calculations have been done for spherical as well as for quadurpole $\beta_{2}$ (both static and dynamic) deformed nuclei with optimum orientations and static hexadecapole $\beta_{4}$ nuclei with compact orientations. It is to be noted that, the rotational energy of ${ }^{214} \mathrm{Rn}^{*}$ is marginally influenced with the inclusion of deformation and orientation effects as shown for $I_{\mathrm{S}}$ limit (see inset of Fig. 1(a)). The higher order deformations contribute more towards $V_{\text {rot }}$ followed by static, dynamic and then spherical nuclei. It is important to note here that, $V_{\text {rot }}$ is decreasing with increase in inter nuclear distance between the interacting nuclei, independent of the choice of deformations and orientation effects. After that, the variation of both modes of the moment of inertia is observed as a function of fragment mass $A_{2}$ at $\mathrm{T}=1.463 \mathrm{MeV}$ using $\beta_{2}$-deformations. It is 


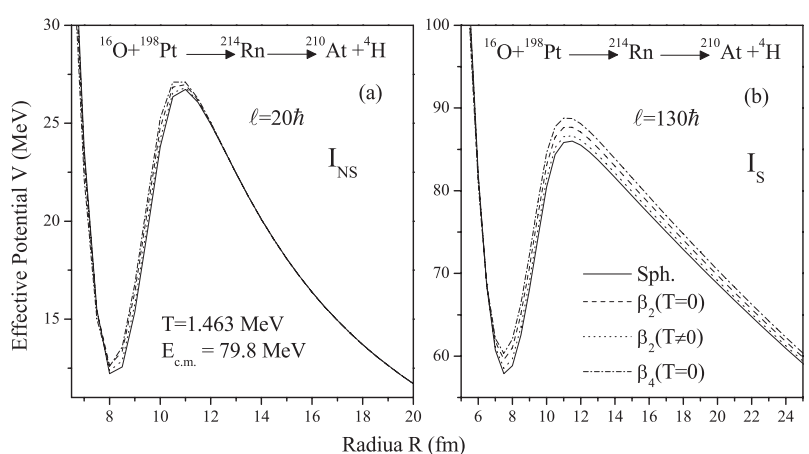

Figure 2. The effective potential $V(\mathrm{R})$ at fixed $\ell$-value of Spherical, static $\left(\beta_{2}(0)\right)$ and dynamic quadurpole deformation $\left(\beta_{2}(\mathrm{~T})\right)$ and static hexadecapole deformation $\beta_{4}(0)$ at $E_{\mathrm{c} . \mathrm{m} .}=$ $79.8 \mathrm{MeV}$ (a) Non-sticking limit of moment of inertia $I_{\mathrm{NS}}$ (b) Sticking limit of moment of inertia $I_{\mathrm{S}}$ for ${ }^{16} \mathrm{O}+{ }^{198} \mathrm{Pt}$ incoming channel.

observed that, the centrifugal potential for light fragments is more within both $I_{S}$ as well as $I_{\mathrm{NS}}$ limits and start decreasing with the increase in mass of decaying fragments as shown in Fig. 1(b). It may be noted that $V_{\text {rot }}$ for $I_{\mathrm{S}}$ is plotted on left vertical scale and that of $I_{\mathrm{NS}}$ on the right vertical scale of Fig. 1(b). Apparently magnitude of $V_{\text {rot }}$ $\left(I_{\mathrm{NS}}\right)$ is much higher as compared to that for $V_{\text {rot }}\left(I_{\mathrm{S}}\right)$.

Since, change in $V_{\text {rot }}$ will bring the corresponding change in the total interaction potential $V_{\mathrm{T}}$ (Eq. (1)) also, so to investigate further the effect of $I_{\mathrm{S}}$ and $I_{\mathrm{NS}}$ limits of moment of inertia, the behavior of total interaction potential is studied as a function of internuclear distance R. Figure 2(a) and Fig. 2(b) shows the variation of scattering potential for the use of $I_{\mathrm{NS}}$ and $I_{\mathrm{S}}$ approaches respectively at minimum energy $E_{\mathrm{c} . \mathrm{m} .}=79.8 \mathrm{MeV}$ in reference to the decay of ${ }^{214} \mathrm{Rn}^{*} \rightarrow{ }^{210} \mathrm{At}+{ }^{4} \mathrm{H}$ reaction for spherical, $\beta_{2}(0), \beta_{2}(\mathrm{~T})$ and $\beta_{4}(0)$ choices of decaying fragments. Figure 2(a) illustrate that at same $\ell$-value i.e. $\ell=20 \hbar$, while using $I_{\mathrm{NS}}$ limit, the barrier characteristics get modified marginally with spherical, $\beta_{2}(0), \beta_{2}(\mathrm{~T})$, and within $\beta_{4}(0)$ deformations. On the other hand, significant variation is seen for all the considered deformations while using $I_{\mathrm{S}}$ limit as shown in Fig. 2(b) plotted at $\ell=130 \hbar$. This further emphasize that, the barrier characteristics are influenced significantly by the choice of moment of inertia, deformation and orientation effects.

It is important to note that, barrier characteristics are major ingredient of the model, which are affected by choice of the moment of inertia, static, dynamic and higher order deformations, thereby affect the fragmentation path of decaying fragments. Therefore, a comparative decay analysis of spherical, $\beta_{2}(0), \beta_{2}(\mathrm{~T})$ and $\beta_{4}(0)$ choices for both $I_{\mathrm{S}}$ and $I_{\mathrm{NS}}$ moment of inertia may impart useful information in context of dynamical behavior of ${ }^{16} \mathrm{O}+{ }^{198} \mathrm{Pt}$ reaction.

The left side panel of Fig. 3 shows the fragmentation path of ${ }^{214} \mathrm{Rn}^{*} \mathrm{CN}$ within $I_{\mathrm{NS}}$ limit, whereas on right side panel fragmentation path within $I_{\mathrm{S}}$ limit is plotted at fixed center of mass energy i.e., $E_{\text {c.m. }}=79.8 \mathrm{MeV}$. At $\ell=0$, i.e., in the absence of centrifugal potential, the fragmentation path of decaying fragments is same for both $I_{\mathrm{NS}}$ and $I_{\mathrm{S}}$ approaches as expected. However, at $\ell_{\max }$, the fragmentation path changes significantly in going from light particle emission to fusion-fission

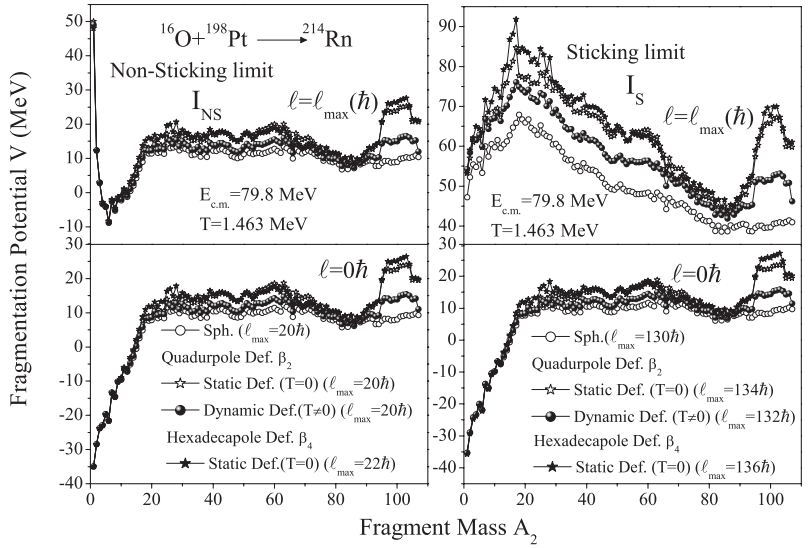

Figure 3. Fragmentation potential $V(\mathrm{MeV})$ as a function of fragment mass $A_{2}$ at fixed $E_{\text {c.m. }}$ for plotted at extreme $\ell$-values, with non-sticking moment of inertia shown in left side panel and sticking moment of inertia shown in right side panel, for spherical, static and dynamic deformation upto quadurpole $\left(\beta_{2}\right)$ alone and hexadecapole $\left(\beta_{4}\right)$ deformed fragments.

(ff) region. For comparison of $I_{\mathrm{NS}}$ and $I_{\mathrm{S}}$ limit of moment of inertia, we observe the following: (i) in $I_{\mathrm{NS}}$ approach, on going from $\ell=0$ to $\ell_{\max }$, the fragmentation behavior is quite similar, except for the light mass region $A_{2} \leq 6$, independent of the choice of deformations and orientations effects. However, the fission region is greatly influenced with the inclusion of temperature dependent and independent deformations. (ii) In $I_{S}$ approach, on going from $\ell=0$ to $\ell_{\max }$, the fragmentation path changes significantly. At lower $\ell$ - values, evaporation residue are dominant decay mode, however, at $\ell_{\max }$, fusion-fission fragments start contributing significantly. The structural behavior of fragments with $\beta_{2}$-static and $\beta_{4}$-static deformations is similar, whereas, for spherical and dynamic $\beta_{2}$-deformations, the fragmentation potential show much lower magnitude with a fare amount of difference in structure.

Here, we have studied both the evaporation residue $\left(A_{2} \leq 4\right)$ and the fusion-fission $\left(\frac{A_{\mathrm{CN}}}{2} \pm 20\right)$ decay modes of ${ }^{214} \mathrm{Rn}^{*}$ compound system. The experimental data is reproduced by using spherical, static, dynamic quadurpole and static hexadecapole deformations. The calculations are done by using only parameter of the model known as neck-length parameter " $\Delta \mathrm{R}$ ", which caters to the relative separation distance between the two fragments or clusters at compound nuclear stage. Table 1 shows the evaporation residue and fusion-fission cross-sections with in the sticking and the non-sticking approaches of moment of inertia. It is anticipated from the calculations that, both evaporation residue and fusion-fission cross-sections are nicely reproduced by $I_{\mathrm{S}}$ approach, except at highest energy for fusion-fission cross-section, which is thereby fitted by the inclusion of static higher order deformations i.e. $\beta_{4}(0)$. However $I_{\mathrm{NS}}$ approach seems suitable only for addressal of evaporation residue data.

The above observation favors the use of moment of inertia in the sticking limit $I_{\mathrm{S}}$ with the inclusion of higher order of deformation $\beta_{4}(0)$ for calculating fission excitation functions, whereas for evaporation residue channel cross-sections, both approaches seem equally probable. In future work, we intend to test the effect of other available moment of inertia on different decay modes of $\mathrm{Rn}$ compound nucleus. 
Table 1. The evaporation residue and fusion-fission cross-sections for ${ }^{214} \mathrm{Rn}^{*}$ system, calculated for different moment of inertia using the DCM at different $E_{\text {c.m. }}$.'s for static, dynamic deformations up to quadurpole $\left(\beta_{2}\right)$ alone and static hexadecapole deformation $\left(\beta_{4}(0)\right)$, compared with the experimental data $[12,13]$.

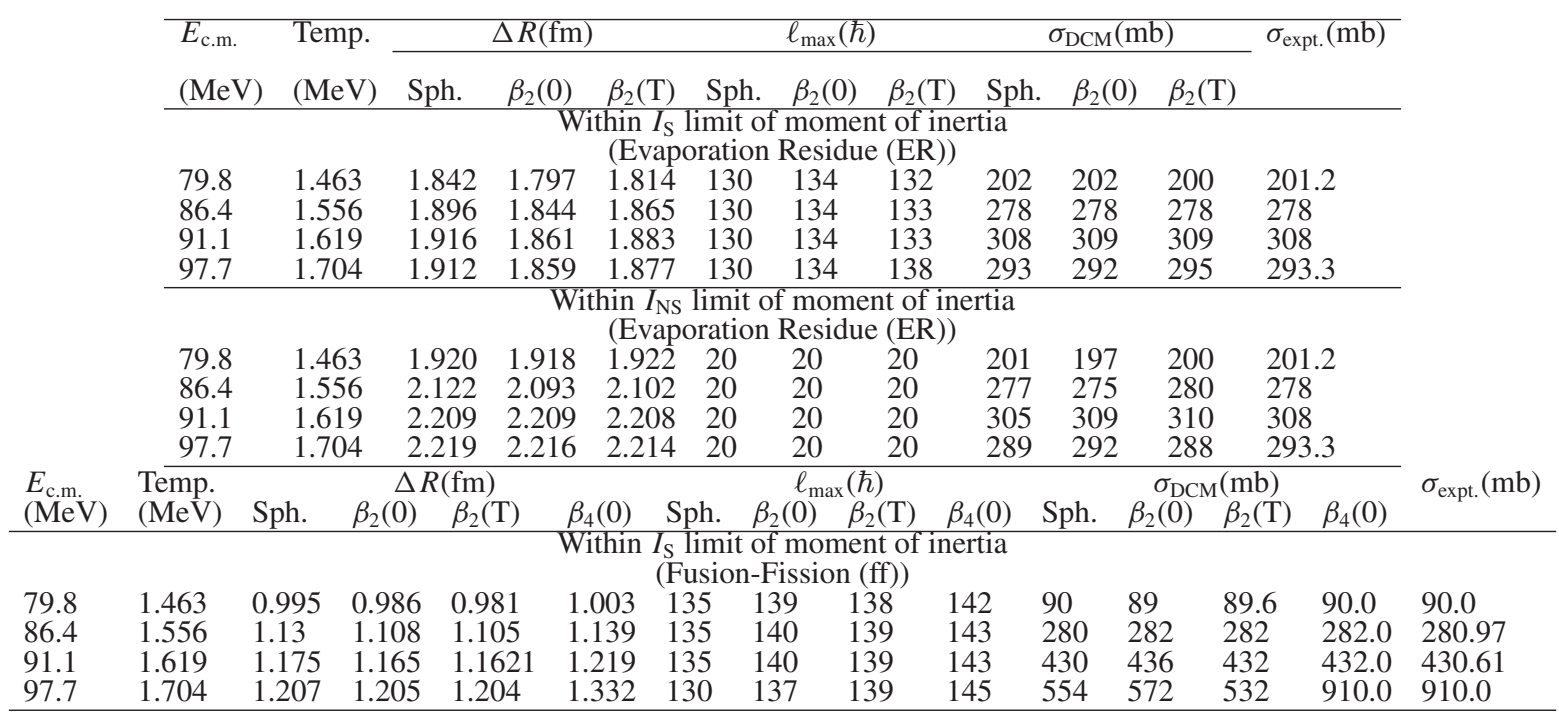

\section{Summary}

We have investigated the role of sticking and nonsticking limit of moment of inertia via barrier profile and fragmentation path of ${ }^{214} \mathrm{Rn}^{*}$ compound nucleus formed in ${ }^{16} \mathrm{O}+{ }^{198} \mathrm{Pt}$ reaction. The fragmentation path changes significantly while going from $I_{\mathrm{NS}}$ to $I_{\mathrm{S}}$ approach. Further, we have studied the role of deformations in the decay of ${ }^{214} \mathrm{Rn}^{*}$ nucleus in which we have observed that spherical and $\beta_{2}(\mathrm{~T})$ deformation behave alike whereas static $\beta_{2}(0)$ and $\beta_{4}(0)$ deformations behave similar in deciding the fragmentation path of decaying fragments. Within the framework of DCM, evaporation residue and fusionfission cross-sections are calculated using both the limits of moment of inertia. It is observed that evaporation residue cross-sections are nicely reproduced by both $I_{\mathrm{NS}}$ and $I_{S}$ approaches by taking into account all type of deformations, however, fusion-fission data show decent agreement only after the inclusion of $\beta_{4}(0)$ deformations within $I_{\mathrm{S}}$ approach.

Work is supported by DST, Govt. of India, Ref. No. EMR/2016/000008.

\section{References}

[1] M. Cavinato, A. Stefanini, Heavy ion fusion (1994)

[2] J. Błocki, J. Randrup, W. Światecki, C. Tsang, Ann. Phys. 105, 427 (1977)

[3] B.V. Derjaguin, Colloid \& Polymer Science 69, 155 (1934)

[4] I. Dutt, R.K. Puri, Phys. Rev. C 81, 064609 (2010)

[5] D. Jain, R. Kumar, M.K. Sharma, Nucl. Phys. A 915, 106 (2013)

[6] B. Singh, M.K. Sharma, R.K. Gupta, Phys. Rev. C 77, 054613 (2008)
[7] G. Kaur, N. Grover, K. Sandhu, M.K. Sharma, Nucl. Phys. A 927, 232 (2014)

[8] K. Sandhu, M.K. Sharma, A. Kaur, R.K. Gupta, Phys. Rev. C 90, 034610 (2014)

[9] R.K. Gupta, R. Kumar, N.K. Dhiman, M. Balasubramaniam, W. Scheid, C. Beck, Phys. Rev. C 68 , 014610 (2003)

[10] R.K. Gupta, M. Manhas, W. Greiner et al., Journal of Physics G: Nucl. Part. Phys. 36, 115105 (2009)

[11] G. Sawhney, R. Kumar, M.K. Sharma, Phys. Rev. C 86, 034613 (2012)

[12] R. Sandal, B. Behera, V. Singh, M. Kaur, A. Kumar, G. Kaur, P. Sharma, N. Madhavan, S. Nath, J. Gehlot et al., Phys. Rev. C 91, 044621 (2015)

[13] R. Sandal, B. Behera, N. Madhavan, S. Nath, J. Gehlot, A. Jhingan, K. Golda, H. Singh, V. Singh, A. Kumar et al., Evaporation residue excitation function measurement for the 16, $18 \mathrm{O}+198 \mathrm{Pt}$ reactions, in Proc. DAE Symp. Nucl. Phys. 57, 532 (2012)

[14] R. Gupta, Clusters in nuclei (lecture notes in physics 818 vol. 1) ed c beck (2010)

[15] R.K. Gupta, W. Scheid, W. Greiner, Phys. Rev. Lett 35, 353 (1975)

[16] R.K. Gupta, J. Phys. G: Nucl. Part. Phys 31, 631 (2005)

[17] R.K. Gupta, M. Manhas, W. Greiner, Phys. Rev. C 73, 054307 (2006)

[18] N. Davidson, S. Hsiao, J. Markram, H. Miller, Y. Tzeng, Phys. Lett. B 315, 12 (1993)

[19] W.D. Myers, W.J. Swiatecki, Nucl. Phys. 81, 1 (1966)

[20] A. Jensen, J. Damgaard, Nucl. Phys. A 203, 578 (1973)

[21] M. Münchow, W. Scheid, Nucl. Phys. A 468, 59 (1987)

[22] M. Münchow, W. Scheid, Phys. Lett. B 162, 265 (1985) 